\title{
Germ cell specification in mice: signaling, transcription regulation, and epigenetic consequences
}

\author{
Mitinori Saitou ${ }^{1,2,3}$ and Masashi Yamaji ${ }^{1,2,3}$ \\ ${ }^{1}$ Department of Anatomy and Cell Biology, Graduate School of Medicine, Kyoto University, Yoshida-Konoe-cho, \\ Sakyo-ku, Kyoto 606-8501, Japan, ${ }^{2}$ Laboratory for Mammalian Germ Cell Biology, RIKEN Center for Developmental \\ Biology, 2-2-3 Minatojima-Minamimachi, Chuo-ku, Kobe 650-0047, Japan and '3JST, CREST, Yoshida-Konoe-cho, \\ Sakyo-ku, Kyoto 606-8501, Japan
}

Correspondence should be addressed to M Saitou at Laboratory for Mammalian Germ Cell Biology, Department of Anatomy and Cell Biology, Graduate School of Medicine, RIKEN Center for Developmental Biology, JST, CREST, Kyoto University;

Email: saitou@anat2.med.kyoto-u.ac.jp

\begin{abstract}
The specification of germ cell fate in development initiates mechanisms essential for the perpetuation of genetic information across the generations. Recent studies in mice have shown that germ cell specification requires at least three key molecular/cellular events: repression of the somatic program, re-acquisition of potential pluripotency, and an ensuing genome-wide epigenetic reprogramming. Moreover, a signaling and transcriptional principle governing these processes has been identified, raising the possibility of inducing the germ cell fate precisely from pluripotent stem cells in culture. These advances will in turn serve as a basis to explore the mechanism of germ cell specification in other mammals, including humans. The recapitulation of germ cell development in humans in culture will provide unprecedented opportunities to understand the basis of the propagation of our genome, both under normal and diseased conditions. Reproduction (2010) 139 931-942
\end{abstract}

\section{Introduction}

The germ cell lineage is the only lineage that ensures the perpetuation and diversification of the genetic information across the generations in most multicellular organisms. The specification of germ cell fate, which establishes a group of cells as primordial germ cells (PGCs), the origin of both oocytes and spermatozoa, initiates essential events required for this process, and it is therefore fundamental to development, reproduction, and heredity. There appear to exist at least two pathways to the specification of germ cell fate: in one pathway, which is called 'preformation', embryonic cells that inherit maternal determinants from the egg go on to form the germ cell lineage, whereas in the other, which is called 'epigenesis', pluripotent cells formed early in development are induced by signals from adjacent tissues to form the germ cell lineage. In mice, and presumably in all mammals, germ cell fate is induced by 'epigenesis' (Saitou 2009b; Fig. 1). Interestingly, however, in both pathways, the repression of the somatic program manifests as a key event, although the molecules underpinning this event are markedly divergent among organisms (Extavour \& Akam 2003, Seydoux $\&$ Braun 2006). Furthermore, it has become increasingly evident that specified PGCs depend on some of the conserved molecules for their proliferation/survival and further development, indicating the presence of common requirements for this lineage across essentially all the species (Extavour \& Akam 2003, Seydoux \& Braun 2006).

In this article, a brief summary of recent advances in the knowledge of the mechanism of germ cell specification in mice, with particular emphasis on signaling and transcription regulation, and their epigenetic consequences, has been given. Evidence shows that, consistent with the function of the germ cell lineage as the transducer of genetic information, PGC specification is an integration of at least three key events: repression of the somatic program, re-acquisition of potential pluripotency, and an ensuing genome-wide epigenetic reprogramming (Saitou 2009a). More detailed knowledge of the mechanism of PGC specification will provide a critical foundation for induction of the germ cell lineage from pluripotent stem cells in vitro not only in mice but also in other mammals, including humans. These advances will in turn lead to a more detailed mechanistic understanding of germ cell specification (e.g. biochemical analysis of the mechanisms involved in PGC specification), as well as advances in reproductive and regenerative medicine. 

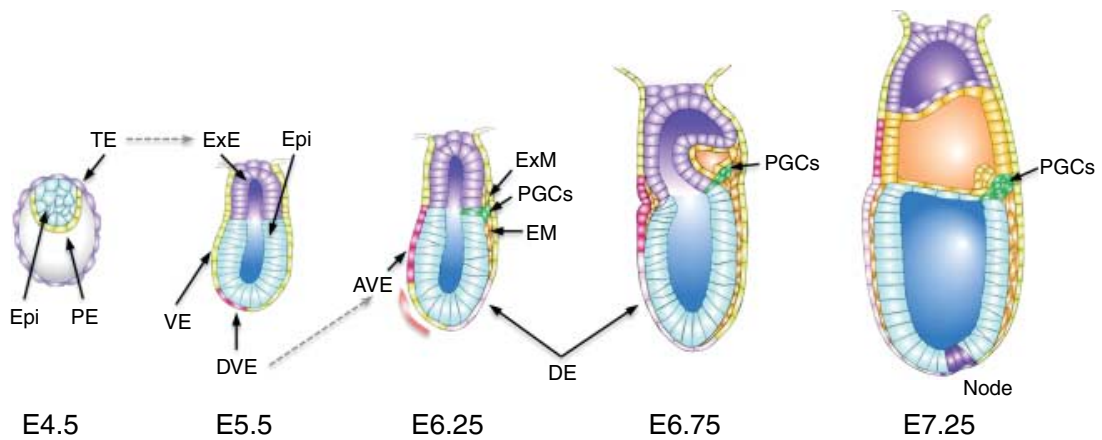

Figure 1 Schematic representation of the emergence of primordial germ cells (PGCs) in the mouse embryo. At the time of implantation ( $\sim E 4.5)$, the blastocyst is composed of three cell types, trophoectoderm (TE, purple), primitive endoderm (PE, yellow), and epiblast (Epi, blue). The TE cells in direct contact with the epiblast proliferate and form a simple epithelial sheet of extraembryonic ectoderm (ExE), which surrounds a small central cavity (the proamniotic cavity). Epiblast cells also undergo cavitation and form a cup-shaped epithelial sheet by E5.5. The epiblast cells have competence to differentiate into all somatic cells as well as germ cells. The initial embryonic patterning including anterior-posterior polarity formation, gastrulation, and germ cell specification is mediated by signalings from the ExE- and PE-derived visceral endoderm (VE) that cover the epiblast (for relevant signals for PGC specification, see text and Fig. 4). DVE, distal visceral endoderm; AVE, anterior visceral endoderm; ExM, extraembryonic mesoderm; EM, embryonic mesoderm; DE, definitive endoderm; PGCs, primordial germ cells.

\section{Transcriptional regulation for PGC specification}

PGCs in mice have been shown to originate from some of the most proximal epiblast cells, to become identifiable during early gastrulation by their characteristic alkaline phosphatase (AP) activity, and to form a distinct AP-positive cluster with $\sim 40$ cells at the base of the incipient allantois in the extraembryonic mesoderm $($ ExM) at around embryonic day (E) 7.25 (early/mid-bud (E/MB) stage; Ginsburg et al. 1990, Lawson \& Hage 1994). Subsequently, and concomitant with an increase in their number, they start to migrate one by one toward the developing hindgut endoderm and move through it. They then exit the endoderm to appear in the mesentery, and at around E10.5 colonize the embryonic gonads, where they proliferate further and initiate a differentiation either into oocytes or spermatozoa depending on their somatic sexes (Bowles \& Koopman 2007).

The precise origin of the germ cell lineage and the mechanism of PGC specification have long been elusive; this has mainly been due to the lack of specific markers that delineate the earliest processes of germ cell specification. For example, it has been known that Pou5f1 (Oct4), a key gene for pluripotency (Niwa 2007), serves as a specific marker for the germ cell lineage, but this is only true after $\sim$ E7.75 (Yeom et al. 1996), much later than PGC specification (PGC specification occurs earlier than E7.0). A series of studies based on single-cell gene expression analysis, including single-cell cDNA microarray analysis, have identified key regulators and global transcription events associated with PGC specification (Saitou et al. 2002, Ohinata et al. 2005, Yabuta et al. 2006, Kurimoto et al. 2008).

B lymphocyte-induced maturation protein 1 (BLIMP1, now known as PRDM1), a PRDI-BF1 and RIZ (PR) domain-containing transcriptional regulator, has long been known as a 'master regulator' of plasma cell differentiation from B-cells (Calame et al. 2003), and has more recently been shown to mark the origin of the germ cell lineage in the epiblast (Ohinata et al. 2005). Prdm1 begins to be expressed in a subset of the most proximal posterior epiblast cells at around E6.25 (pre/no-streak (P/OS) stage). The Prdm1-expressing cells increase in number, move posteriorly, and develop into PGCs with AP activity, Dppa3 (stella) expression, and Hox gene repression at around E7.25 (Figs 1 and 2).

Prdm1-positive cells initially express the Hox genes as well as many genes known to be involved in embryonic development, especially pattern specification and mesoderm induction (including genes such as Hoxa1, Hoxa2, Hoxa3, Hoxb1, Hoxb2, Hoxd1, Hoxd9, Snai1, Tbx3, Tbx6, Mesp1, Sp5, Mixl1, Sall3, Cdx1, Isl1, Ets1, Etv2, Nfkbia, Foxf1, Plxna2, and Smad7), but subsequently downregulate them from around E6.75-E7.0 (late-streak/no-bud (LS/OB) stages; Yabuta et al. 2006, Kurimoto et al. 2008). Conversely, Prdm1-positive cells initially downregulate the expression of genes associated with pluripotency, including Sox2, Nanog, and Zic3, but regain them from around the LS/OB stage (Yabuta et al. 2006, Kurimoto et al. 2008). Therefore, the PGC precursors appear to be initially propelled toward a somatic mesodermal fate, but then regain their potentially pluripotent nature.

At the E/MB stages, compared to their somatic neighbors, which would share a common origin, PGCs up- and downregulate $\sim 500$ and $\sim 330$ genes ('germ cell specification' and 'somatic program' genes) respectively (Kurimoto et al. 2008). Accordingly, the specification genes include those for 'germ cell development' in the Gene Ontology (GO) functional annotation (Prdm1, Dppa3, Ifitm3, Dnd1, and Kit). They do not include, however, a particularly large number of genes in the other functional categories, suggesting that the PGCspecific genes consist of a unique set of genes. The specification genes include transcriptional regulators 

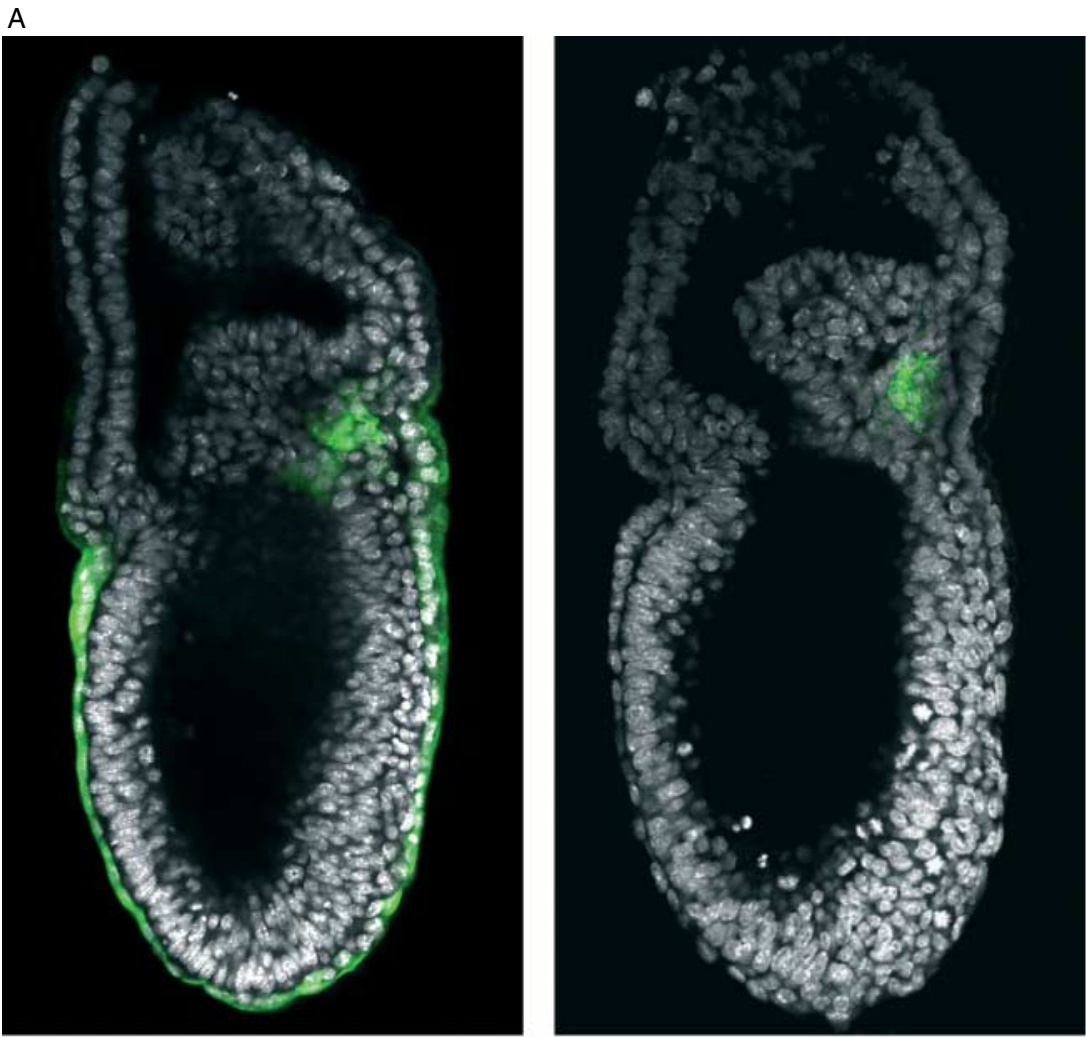

B

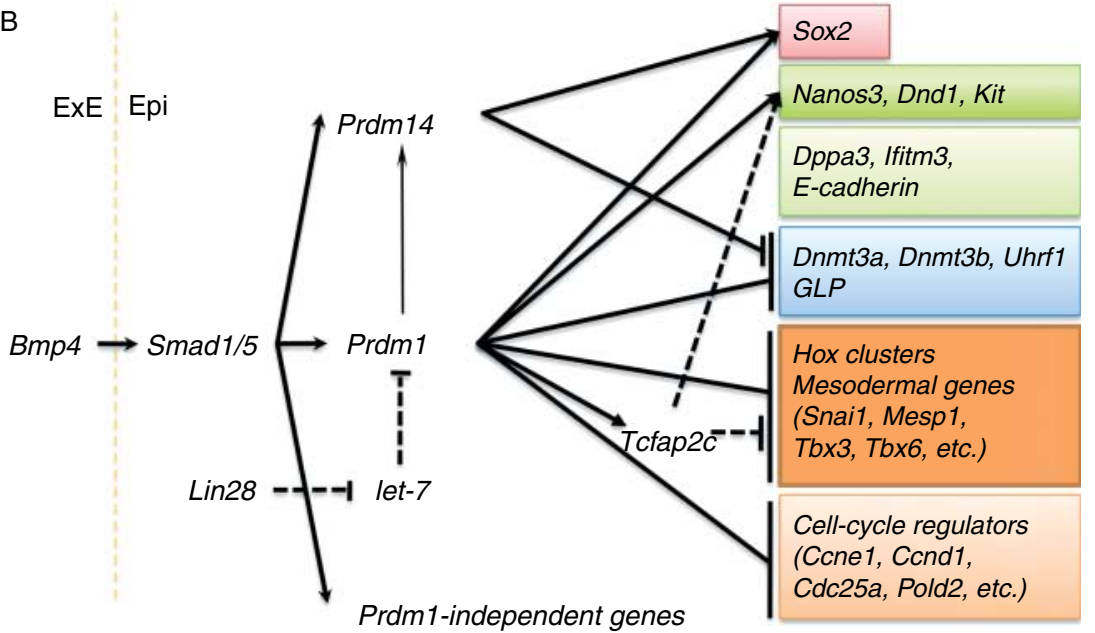

Figure 2 A model of PGC specification orchestrated by the two PR domain-containing proteins, PRDM1 and PRDM14. (A) Expression of Prdm1 (left) and Prdm14 (right) in the LS stage embryo visualized by the Prdm1-mVenus and $\operatorname{Prdm} 14-\mathrm{mVenus}$ reporters respectively. $\operatorname{Prdm} 1$ is expressed in the nascent PGC precursors emerging from the most proximal part of the posterior epiblast as well as in the visceral endoderm. Prdm14 is exclusively expressed in the germ cell lineage and pluripotent cell lines. (B) A summary of genetic pathways for PGC specification. See text for details. Black arrows and black lines with terminal bars indicate genetic pathways for activation and for repression respectively, as demonstrated by in vivo experiments. Dotted arrows and dotted lines with terminal bars indicate genetic pathways for activation and for repression respectively, as proposed based on in vitro experiments. such as Prdm1, Prdm14, Sox3, Tcfap2c, Elf3, Elk1, Isl2, Mycn, Klf2, Fiat, Sp8, Smad3, Sox2, Nanog, Zic3, Tcfe3, Epc1, Six4, and Eya3, some of which should have critical functions in PGC specification (see below). In addition to the genes associated with pattern specification and mesoderm development, the somatic genes include many cell cycle-associated genes, such as S-phase promoting factors (e.g. Ccne1, Ccnd1, Cdc25a, Cdc6, Pold2, E2F3, and Myc) and regulators of DNA methylation (e.g. de novo DNA methyltransferases (Dnmt3a and Dnmt3b) and Uhrf1, the essential factor for the recruitment of Dnmt1 to replication foci;
Bostick et al. 2007, Sharif et al. 2007). The precise gene expression signature for the established PGCs provides a basis for the analysis of the function of each gene up- or downregulated in PGCs.

\section{Key regulators for PGC specification}

Most notably, PRDM1 has been found to be one of the most critical regulators for PGC specification (Ohinata et al. 2005, Vincent et al. 2005, Kurimoto et al. 2008; Fig. 2). In the absence of PRDM1 activity, AP-positive PGC-like cells are formed, but they are smaller in 
number, form aberrant aggregates, and do not show migration toward the hindgut endoderm. Single-cell cDNA microarray analysis has shown that the Prdm1deficient PGC-like cells fail to repress almost all the somatic genes (Kurimoto et al. 2008). In contrast, while they exhibit severe impairment in the upregulation of highly PGC-specific genes, they more or less upregulate approximately half of the specification genes $(\sim 250$; Kurimoto et al. 2008). However, a rigorous quantitative comparison of single-cell gene expression levels and frequencies of the specification genes between the wild-type and Prdm1-deficient cells shows that PRDM1 does exert positive and quantitative effects on the upregulation and concerted expression of many of the highly PGC-specific genes (Kurimoto et al. 2008). Consistently, the effect of Prdm1 on PGC specification is dose dependent, with fewer PGCs specified in its heterozygous mutants (Ohinata et al. 2005, Vincent et al. 2005, Robertson et al. 2007). These observations indicate that, while Prdm1 is essential for repressing all the somatic genes, it may also be important for creating an epigenetic state for the expression of highly PGCspecific genes.

PRDM14, another PR domain-containing protein, is also a critical regulator for PGC specification (Yamaji et al. 2008). Prdm14 is evolutionally conserved at least in vertebrates. It has been reported that in humans, Prdm14 is upregulated in some cancers (Hu et al. 2005, Nishikawa et al. 2007) and also in embryonic stem (ES) cells (Assou et al. 2007, Tsuneyoshi et al. 2008). In mice, PRDM14 starts to be expressed in the morula and continues to be expressed in the inner mass cells of the blastocysts, but this expression is transient and disappears quickly by $\sim$ E5.0. Subsequently, its expression re-initiates specifically in PGC precursors at around E6.5 (P/ES stage) and continues exclusively in PGCs until about $\sim$ E13.5-E14.5 in both males and females. In contrast to Prdm1, Prdm14 does not appear to be expressed in any cell types other than PGCs. In the absence of PRDM14, AP-positive PGC-like cells are formed, and they repress the Hox genes, but fail to upregulate SOX2 (Yamaji et al. 2008). They also fail to undergo proper genome-wide epigenetic reprogramming (see below), partly due to a failure to repress GLP, and eventually disappear perhaps due to the failure to proliferate. A global transcriptome analysis of Prdm14-deficient PGC-like cells shows that they acquire characteristics somewhat similar to Prdm1-positive, Hoxb1-negative LS/OB stage cells, but do not fully mature into PGCs at the E/MB stage (M Saitou, unpublished observation). Importantly, the initial expression of Prdm14 in PGCs is Prdm1 independent, but its subsequent maintenance and/or upregulation is strictly dependent on Prdm1. Thus, PRDM1 and PRDM14 are the two major transcriptional regulators orchestrating the birth of the germ cell lineage in mice (Fig. 2).
Transcription factor TCFAP2C (also known as AP2 $\gamma$ ), a member of the five closely related transcription factors bearing a characteristic basic helix-span-helix domain (Eckert et al. 2005), also plays a key role for PGC specification (Weber et al. 2010). TCFAP2C seems to be expressed maternally and is detected in all the cells at least up to the blastocyst stage but subsequently becomes confined to the extraembryonic ectoderm (ExE) and the PGCs (Auman et al. 2002, Werling \& Schorle 2002). Epiblast-specific deletion of Tcfap2c shows that, in the absence of Tcfap2c, AP-positive PGC-like cells appear to be formed normally, but their numbers become drastically reduced by E8.0 and they do not show characteristic migration toward the hindgut endoderm (Weber et al. 2010), a phenotype similar to that in Prdm1-deficient embryos. Since Prdm1 expression precedes $T c f a p 2 c$ and Prdm 1 deletion leads to the loss of Tcfap2c upregulation, Tcfap2c would be a critical downstream target of PRDM1 for PGC specification (Fig. 2).

It has recently been suggested that the RNA-binding protein LIN28 may play a role as an upstream regulator for PRDM1 (West et al. 2009). LIN28 is an RNA-binding protein bearing a cold-shock domain and two retroviraltype zinc finger domains of the CCHC type, and has been shown to inhibit the processing and maturation of let-7 miRNA family members (for review see Bussing et al. (2008)). Notably, LIN28 is one of four genes (the others being POU5F1, SOX2, and NANOG) that together suffice to reprogram human fibroblast cells into induced pluripotent stem (iPS) cells (Yu et al. 2007), although the role of LIN28 in this process has yet to be determined. On the other hand, in pathologic Reed-Sternberg cells, let-7a is highly expressed and suppresses PRDM1 by binding to a target site in the PRDM1 3' UTR (Nie et al. 2008). Using an in vitro ES cell differentiation strategy, a study by West et al. (2009) showed that Lin28 is an essential regulator of PGC specification through inhibition of let-7 maturation and consequent induction of Prdm1 (Fig. 2). The precise expression of LIN28 in the epiblast and in the initial phase of PGC specification and whether LIN28 is indeed critical in PGC specification in vivo remain to be determined.

There are a number of genes that are known to play critical roles in the early phase of PGC development, presumably after the PGC fate is established (for review see Saitou (2009b)). It has long been known that the receptor tyrosine kinase Kit and its ligand stem cell factor regulate the migration, proliferation, and/or survival of PGCs (Mintz \& Russell 1957, McCoshen \& McCallion 1975, Buehr et al. 1993, Gu et al. 2009), presumably through the AKT/MTOR/BAX signaling pathway (De Miguel et al. 2002, Stallock et al. 2003, Runyan et al. 2006). Similarly, mutations of RNA-binding proteins such as NANOS3, TIAL1, and DND1 have been known to lead to the loss of germ cells due to the failure of survival and/or proliferation of PGCs (Beck et al. 1998, 
Tsuda et al. 2003, Youngren et al. 2005). It is also of note that hypoxia-inducible factor-2a has been shown to play a critical role for PGC specification and/or early PGC development, presumably by regulating POU5F1 expression in PGCs (Covello et al. 2006). The precise mechanisms of the actions of these molecules in PGCs and the modes of their regulation by the key specification genes, such as Prdm1 and Prdm14, remain to be investigated. It will also be important to clarify the precise roles of key pluripotency genes such as Pou5f1, Sox2, and Nanog for PGC specification (Okamura et al. 2008), although POU5F1 and NANOG have been shown to be critical to the survival of PGCs at relatively later stages, i.e. E9.5 (Kehler et al. 2004, Chambers et al. 2007, Yamaguchi et al. 2009).

\section{Epigenetic reprogramming following PGC specification}

PGCs have been shown to undergo extensive epigenetic reprogramming, including genome-wide DNA demethylation, erasure of parental imprints, and re-activation of the inactive X-chromosome (for review see Sasaki \& Matsui (2008)). However, the mechanisms underlying these events have long been elusive. A precise understanding of the molecular mechanisms leading to PGC specification, the crucial onset of the entire epigenetic reprogramming process in the germ cell lineage, will be a key to addressing this issue (Figs 2 and 3).

It has been shown that Prdm1-positive PGC precursors at around E6.75 (early/mid-streak (E/MS) stage) bear genome-wide epigenetic modifications (di- and trimethylation of $\mathrm{H} 3$ lysine 4 (H3K4me2 and me3) and acetylation of $\mathrm{H} 3$ lysine 9 (H3K9ac; active modifications), mono-, di-, and trimethylation of $\mathrm{H} 3$ lysine 9 (H3K9me1, me2, and me3), and di- and trimethylation of $\mathrm{H} 3$ lysine 27 (H3K27me2 and me3; repressive modifications)), apparently indistinguishable from their somatic neighbors (Seki et al. 2007). Subsequently, however, from around E7.75 onwards, PGCs that have started their migration begin to show the genome-wide reduction of the two major repressive modifications, DNA methylation and H3K9me2 (Seki et al. 2005, 2007). Although the mode of reduction of genome-wide DNA methylation in migrating PGCs has not yet been precisely analyzed, the global reduction of $\mathrm{H} 3 \mathrm{~K} 9 \mathrm{me} 2$ in migrating PGCs seems to be a progressive, cell-by-cell process, with nearly all the PGCs exhibiting low H3K9me2 levels by E8.75 (Seki et al. 2007).

As discussed earlier, upon PGC specification, PRDM1 and PRDM14 are directly or indirectly involved in the repression of key machineries for both de novo and maintenance DNA methylation, and in the repression of the histone methyltransferase GLP, an essential enzyme for genome-wide H3K9me1 and me2 in embryonic development (Tachibana et al. 2005). On the other hand, although some of the JmjC domain-containing histone lysine demethylases (see review, Klose et al. (2006) and Shi (2007)) are indeed expressed in PGCs, none of them are specific to PGCs (i.e. similarly expressed in somatic neighbors). These observations support the idea that the specific repression of active enzymes in PGCs may be a key to understanding the genome-wide reduction of DNA methylation and $\mathrm{H} 3 \mathrm{~K} 9 \mathrm{me} 2$. Since there is experimental evidence that supports the presence of active mechanisms for the erasure of epigenetic modifications (Hajkova et al. 2002, Seki et al. 2007), there should exist some intricate interplay between active and passive mechanisms (DNA methylation being reduced passively by cell division, especially after E9.0 (see below), and H3K9me1/2 being reduced either through a turnover of methyl groups or a replacement of the entire $\mathrm{H} 3$ molecule) in PGCs for the epigenetic reprogramming.

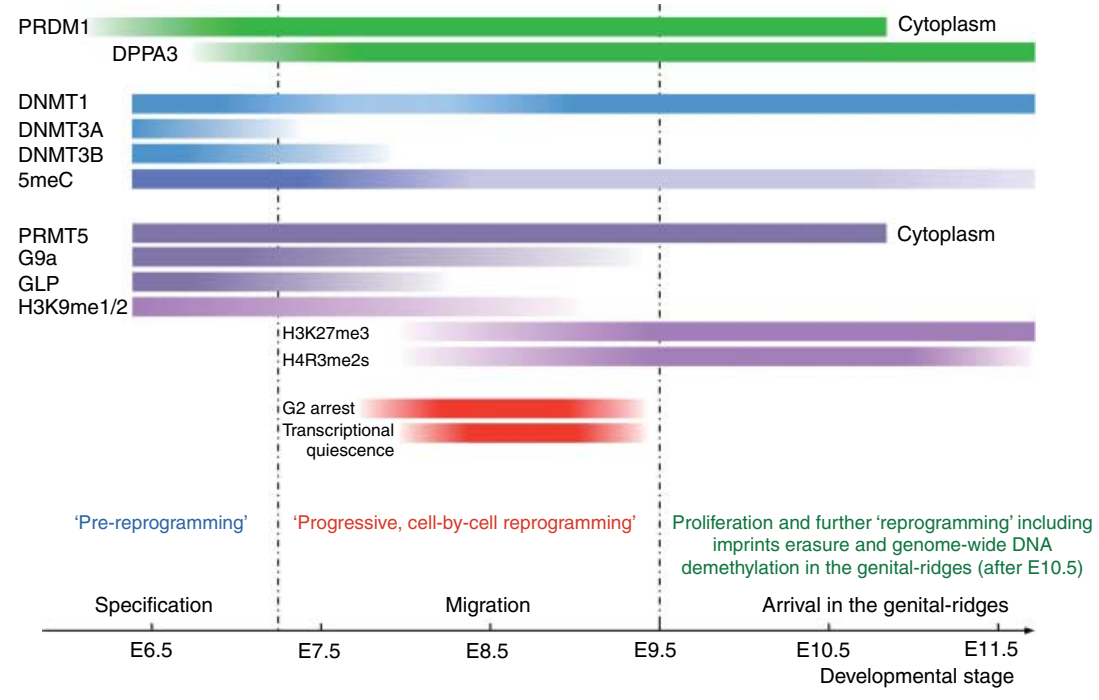

Figure 3 A summary of the genome-wide epigenetic reprogramming revealed by immunohistochemistry in migrating PGCs. The established PGCs that start migration undergo a genome-wide epigenetic reprogramming, which includes genome-wide reduction of DNA methylation and $\mathrm{H} 3 \mathrm{~K} 9 \mathrm{me} 2$, and genome-wide upregulation of H3K27me3 and H4R3me2. PGCs repress active enzymes for repressive epigenetic modifications (DNA methylation and $\mathrm{H} 3 \mathrm{~K} 9 \mathrm{me} 1 / 2$ ) prior to the reprogramming, become arrested at the $\mathrm{G}_{2}$ phase of the cell cycle, and turn off RNA polymerase II-based transcription during the reprogramming. See text for details. 
On the other hand, genome-wide H3K27me3, another repressive modification mediated by the polycomb repressive complex 2 (PRC2), becomes upregulated in migrating PGCs from around E8.25 onwards in a progressive, cell-by-cell manner, with nearly all the PGCs showing high H3K27me3 levels by E9.5 (Seki et al. 2005, 2007). EZH2, EED, and SUZ12, the three core components of the PRC2 complex that mediate the trimethylation of H3K27 (Cao \& Zhang 2004), are expressed at similar levels both in the PGCs and their somatic neighbors by at least E8.25 (Yabuta et al. 2006, Kurimoto et al. 2008). It may therefore be the case that the preceding reduction of DNA methylation and $\mathrm{H} 3 \mathrm{~K} 9 \mathrm{me} 1 / 2$ would play a role in the upregulation of H3K27me3.

Notably, a majority of migrating PGCs $(\sim 60 \%)$ from around E8.0 to around E9.0, a key period of epigenetic reprogramming, are shown to be in the $\mathrm{G}_{2}$ phase of the cell cycle (Seki et al. 2007). PRDM1 may be a key factor for the $\mathrm{G}_{2}$ arrest, since it is involved in the repression of S-phase promoting factors (e.g. Ccne1, Cond1, Cdc25a, Cdc6, Pold2, E2F3, and Myc) (see above) (Kurimoto et al. 2008). Moreover, concomitant with this period, PGCs seem to transiently pause their global transcription by RNA polymerase II (Seki et al. 2007). These observations indicate that PGC specification creates a unique cellular state for the epigenetic reprogramming: PGCs repress key active epigenetic enzymes, are arrested at the $\mathrm{G}_{2}$ phase of the cell cycle, and pause Pol II-based transcription.

Furthermore, it has been reported that migrating PGCs upregulate the symmetrical dimethylation of histone $\mathrm{H} 4$ arginine 3 (H4R3me2), which appears to be conferred by a protein arginine methyltransferase, PRMT5 (Ancelin et al. 2006). PRMT5 catalyzes $\omega-N^{G}, N^{\prime G}$-symmetric dimethylation of arginine residues, in a variety of target proteins, including histone $\mathrm{H} 4, \mathrm{H} 2 \mathrm{~A}$, and $\mathrm{H} 3$, and the spliceosomal proteins SmD1, SmD3, and SmB/B' (Bedford \& Richard 2005). Ancelin et al. have shown that PRDM1 forms a complex with PRMT5 in cultured cell lines and co-localizes with PRMT5 in the nuclei of migrating PGCs. The co-localization of PRDM1 and PRMT5 in the PGC nuclei appears to be seen only between E8.5 and E10.5, and after E11.5, both proteins seem to translocate into the cytoplasm, coincident with the downregulation of $\mathrm{H} 4 \mathrm{R} 3 \mathrm{me} 2$ in the PGC nuclei. The function of PRMT5 in PGC specification and the significance of the putative PRDM1/PRMT5 complex in PGCs remain to be explored.

In sum, it has now become evident that PGC specification involves a complex genetic program that leads to a genome-wide epigenetic reprogramming (Figs 2 and 3). Further clarification of the functions of the many genes involved in PGC specification, including Prdm1, Prdm14, and Tcfap2c, is therefore critical to understanding the initial setup and the consequences of the epigenetic reprogramming in the germ cell lineage.

\section{A signaling principle for PGC specification}

It has long been known, through gene knockout studies, that bone morphogenetic protein (BMP) signals are essential for the generation of AP-positive PGCs from the epiblast: BMP4 and BMP8B emitted from the ExE and BMP2 emitted apparently from the visceral endoderm (VE), as well as ALK2, a type I receptor for BMP signaling and the signal transducers known as SMADs (SMAD1, 4, and 5), are critical for the generation of AP-positive PGCs (for review see Saitou (2009b)). However, due to the absence of sensitive markers for the detection of nascent PGCs, the precise mechanism through which the BMP signals generate the germ cell lineage has been elusive. Hence, numerous questions remain unanswered: what are the direct target cells of BMPs, which receptor complexes do the BMPs use, what are their downstream target genes, how do the different BMPs cooperate to induce PGCs, and are there any secondary signals that are required for PGC specification?

The identification of Prdm1 and Prdm14 as genes that specifically mark the very onset of PGC specification in the epiblast and the generation of robust reporters for their expression have made it possible to investigate the precise roles of signaling molecules in PGC specification. By analyzing the expression of Prdm1 and Prdm14 using their expression reporters in various signaling mutants and in isolated epiblasts cultured under serum-free, defined conditions, a recent study has succeeded in constructing an integrated model regarding the roles of signaling activities that confer the germ cell fate to the epiblast cells (Yamaji et al. 2008, Ohinata et al. 2009).

In all the mutants for Bmp4,Bmp8b, or Smad1, the expression of Prdm1 and Prdm14 in the epiblasts is severely impaired, indicating that BMP signals are necessary for the earliest step in PGC specification. On the other hand, in mutants for Smad2 or FoxH1, in which nearly all the epiblast cells adopt a posterior fate due to impaired formation of anterior VE (AVE), a signaling center emitting the anteriorization/anti-posteriorization signals (e.g. LEFTY1 against NODAL, DKK1 against WNT, Cerberus-like against BMP, etc.; Arnold \& Robertson 2009), Prdm1 expression expands into all the epiblast cells contacting the ExE, as well as those locating much more distally. This indicates that in the absence of the anteriorizing signals, all the pre-streak stage epiblast cells may adopt the germ cell fate as long as they receive BMP signals from the ExE. Consistently, in vitro epiblast cultures show that in response to BMP4, essentially all the isolated epiblast cells from E5.5 to E6.0 are competent to express Prdm1 and Prdm14, and take on the AP-positive germ cell fate: through the complex of ALK3 (or ALK6) and one of the type II receptors (most likely BMPR2) via SMAD1 and SMAD5, BMP4 induces both Prdm1 and Prdm14 in the epiblast in a dosedependent manner. In addition, BMP2 also induces Prdm1 and Prdm14 in the epiblast, although less 
efficiently than BMP4. Notably, this study shows that BMP8B does not directly induce Prdm1 and Prdm14 in the epiblast but rather controls AVE development to an appropriate level: in Bmp8b mutants, AVE becomes enlarged and apparently emits an extra amount of anteriorizing signals which prevent the BMP4 signaling. These findings show that the three BMP molecules BMP4, BMP8B, and BMP2 cooperate during early gastrulation to endow the most proximal posterior epiblast cells with a sufficient level of BMP-SMAD signaling for their adoption of the germ cell fate (Fig. 4).

A previous report has shown that BMP4 signaling through the VE is essential for PGC specification (de Sousa Lopes et al. 2004). In contrast, the study by Ohinata et al. has shown that BMP4 signals directly to the epiblast cells. The culture used in the previous study included serum and feeder cells, some unidentified factor(s) which could have complicated the experimental outcome. Furthermore, for detecting PGCs, the previous study depended almost solely on AP staining, which is not a strict criterion to identify PGCs in culture. These points may explain the discrepancy of the conclusions between the two studies.

It is also of note that only the epiblast cells from E5.5 to E6.25 are competent to express Prdm1 and Prdm14 and subsequently take on the AP-positive germ cell fate in response to BMP4 (Ohinata et al. 2009): epiblast cells at E5.25 show neither Prdm1 nor AP activity, whereas those later than E6.5 exhibit Prdm1 but fail to show AP activity and Dppa3 in response to BMP4, indicating that the epiblast cells alter their responsiveness to BMP4 in a developmental stage-dependent manner. Importantly, this study shows that WNT3, which begins to be expressed in the epiblast at around E5.5 (Liu et al. 1999, Kemp et al. 2005), is a key factor conferring the BMP4 responsiveness to the epiblasts, although the molecular underpinning of the 'competence' and how WNT3 might provide it need to be clarified. On the other hand, the fact that epiblast cells at E6.5 do express Prdm1 in response to BMP4 but do not exhibit AP activity or Dppa3 indicates that PRDM1 alone is not sufficient to confer the germ cell fate. In good agreement with these findings, PRDM1 has been shown to be expressed in multiple cell lineages during development and adulthood, and in many of these cases, it plays a critical role for the lineage specification or maintenance (Horsley et al. 2006, Kallies et al. 2006, Martins et al. 2006, Magnusdottir et al. 2007, Robertson et al. 2007). The epigenetic landscape of the cells in which PRDM1 is induced would therefore be a key to how PRDM1 affects the fate of the cells. Accordingly, the genes controlled by PRDM1 in PGC specification and in other contexts such as epidermal or plasma cell differentiation are very different (Kurimoto et al. 2008).

One of the most significant demonstrations of this study is that under a defined condition essentially with BMP4, a majority of the competent epiblast cells go on to develop into pre-meiotic PGC-like cells after

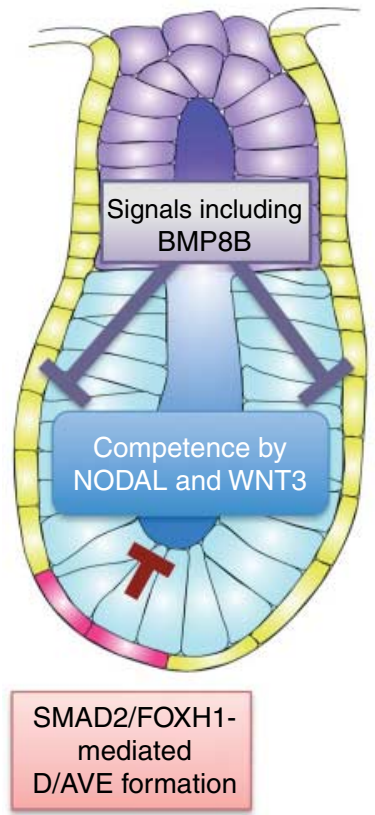

E5.5- 5.75

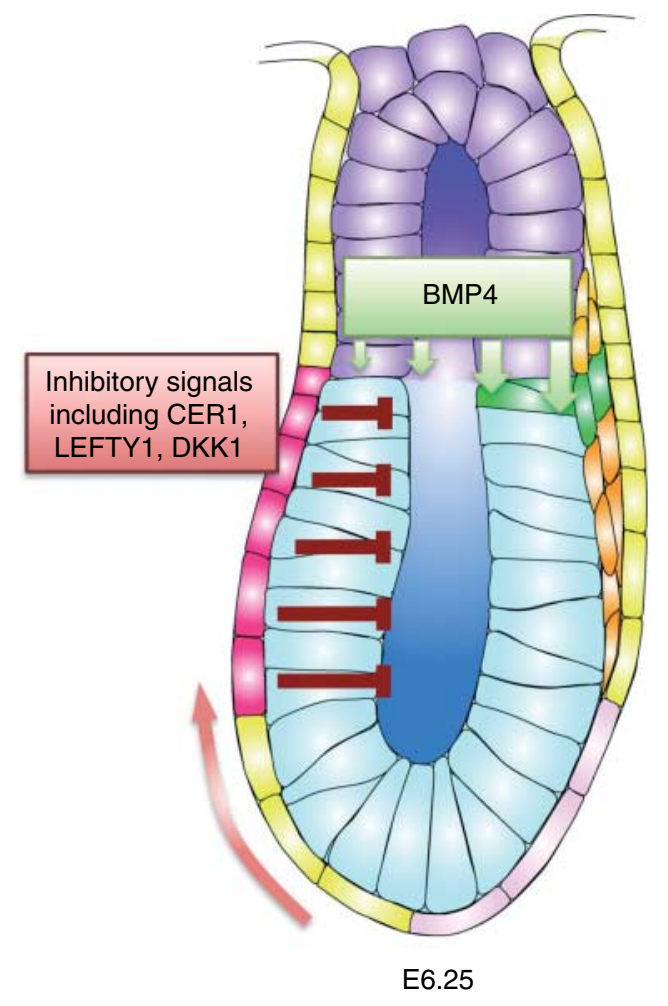

Figure 4 Signaling activities leading to PGC specification in the mouse embryo. At around E5.5, epiblast cells acquire the competence to respond to BMP4 by the activities of NODAL and WNT3. On the other hand, NODAL signaling mediated by SMAD2/FOXH1 specifies DVE cells that start to provide signals against posteriorization. Signals from ExE including BMP8B apparently prevent proximal VE from differentiating to DVE, thus restricting the anti-posteriorization activities. At around E6.0-6.25, DVE moves anteriorly (red arrow) to form AVE, and a subset of the competent epiblast cells that receive the highest level of BMP4 signals from the ExE are specified as PRDM1- and PRDM14-positive PGC precursors. The germ cell competence may be sustained until around the LS stage in the proximal part of posterior epiblast cells. The abbreviations are as shown in Fig. 1. 
84-132 h of culture (Ohinata et al. 2009). The induced PGC-like cells not only show proper gene expression, but also exhibit characteristic epigenetic reprogramming, including the reduction of genome-wide H3K9me2, upregulation of H3K27me3, and erasure of the genomic imprints, an attribute exclusive to PGCs, during the 132-h culture. These findings indicate that PGC specification may be an instructive event that allows a major part of the subsequent development of PGCs to progress in a cell-autonomous manner. Finally, the induced PGC-like cells contribute to functional spermatogenesis when directly transplanted into neonatal germ cell-less testes of $W / W^{v}$ mice or by the gonad reconstruction assay, providing an unequivocal and stringent demonstration of the germ cell fate induction.

\section{PGC specification from pluripotent stem cells in culture}

There have been several reports regarding the derivation of gametes from ES cells in culture (Hubner et al. 2003, Toyooka et al. 2003, Geijsen et al. 2004, Nayernia et al. 2006). However, the generation of fully functional gametes that support normal embryonic development and adult physiology has not yet been achieved. The methodologies in these reports use serum and do not involve directed differentiation toward the germ cell lineage, but rather select rare cells expressing some germ cell markers after prolonged culture for random differentiation. Accordingly, complex processes required for germ cell development in vivo have not been monitored properly.

In recent years, there has been considerable progress in our understanding of the molecular basis of many aspects of germ cell development, including PGC specification, as reviewed here, male versus female germ cell differentiation, meiosis, spermatogonial stem cell biology, spermatogenesis, and oocyte development (for reviews see Bowles \& Koopman (2007), KanatsuShinohara et al. (2008), Edson et al. (2009) and Handel \& Schimenti (2010)). Based on the information we have obtained, for the successful generation of gametes from pluripotent stem cells in culture, it would be essential and perhaps most efficient to recapitulate what happens in vivo from the very beginning (i.e. PGC specification from the epiblast) as precisely as possible and in a step-by-step fashion.

The signaling requirements that are responsible for the expression of key transcriptional regulators for PGC specification from the epiblast have been revealed, which has led to the generation of functional PGC-like cells under a serum-free, defined condition (Ohinata et al. 2009). Several methodologies to induce ES cells into epiblast-like states under a serum-free, defined condition have been reported (for review see
Murry \& Keller (2008)). It would therefore be possible to induce PGC-like cells from pluripotent stem cells, including ES cells and iPS cells (Takahashi \& Yamanaka 2006, Okita et al. 2007, Takahashi et al. 2007, Wernig et al. 2007, Yu et al. 2007, Park et al. 2008), by first differentiating them toward an epiblast-like state.

Recent studies have reported the derivation in culture of pluripotent stem cells from the epiblast, i.e. EpiSCs, which bear properties similar to those of the original epiblast cells, including gene expression and epigenetic states (Brons et al. 2007, Tesar et al. 2007). EpiSCs can be derived from the epiblast cells at E5.5-E6.5 in the presence of activin and basic fibroblast growth factor (FGF). They can also be induced from ES cells by culturing them with activin and bFGF (Guo et al. 2009). These studies have raised the important possibility of inducing PGC-like cells from EpiSCs or EpiSC-like cells cultured under certain modified conditions. Indeed, it has been shown that EpiSCs are a somewhat heterogeneous cell population that includes Prdm1- and Dppa3-positive cells, although these cells are present in very small numbers (the Dppa3-positive cells constitute $0.5 \%$ of the total EpiSC population), and that EpiSCs have many important properties that are similar to those of PGCs, including gene expression, epigenetic states, and differentiation potential (Hayashi \& Surani 2009). The emergence of Dppa3-positive PGC-like cells in the EpiSC culture is blocked by dorsomorphin, an inhibitor of BMP signaling, and enhanced by BMP4 (up to $\sim 1.5 \%$ of cells becoming Dppa3-positive, a similar ratio for the emergence of Dppa3-positive cells in randomly differentiated embryoid bodies; West et al. 2009), indicating that a signaling pathway operating in vivo is indeed responsible for the emergence of PGC-like cells in culture (Hayashi \& Surani 2009). The function of the PGC-like cells has not been definitively demonstrated by transplantation assays. Further modification of the culture conditions would lead to a more efficient generation of PGC-like cells in culture.

Perhaps, one of the most important challenges following a precise recapitulation of PGC specification would be to explore the conditions necessary to support a long-term proliferation of PGCs in vitro. The long-term proliferation of PGCs in turn would provide a foundation for exploring the conditions needed to support further germ cell development in vitro. There have been several attempts to establish such long-term proliferation, but PGCs in culture either go on to undergo apoptosis after several rounds of cell divisions (Dolci et al. 1991, Matsui et al. 1991, Pesce et al. 1993) or dedifferentiate into pluripotent embryonic germ cells (Matsui et al. 1992, Labosky et al. 1994, De Miguel et al. 2002). PGCs in vivo go on to enter the cell cycle arrest at the $\mathrm{G}_{1}$ phase in the male genital ridges or the meiotic prophase in the female genital ridges, both after around E13.5 (McLaren 2003). However, PGCs proliferate actively, especially when they migrate out from the developing hindgut endoderm 
(see above; Seki et al. 2007) and in the genital ridges in earlier stages ( $\sim$ E10.5-E11.5). Understanding of the mechanisms for PGC survival and proliferation would therefore be critical for establishing the conditions for long-term proliferation of PGCs in culture.

\section{Perspectives: implications for PGC development in other mammals, including humans}

In the last decade of intensive research, a major part of the framework for the signaling and transcription in PGC specification in mice has been established. In particular, the identification of PRDM1 and PRDM14 as critical transcriptional regulators for PGC specification has been a key breakthrough. The finding that essentially all the epiblast cells from E5.5 to E6.0 are competent to express PRDM1 and PRDM14 and go on to form PGCs in response to BMP4 constitutes an important basis from which to explore conditions for inducing PGC-like cells directionally from pluripotent stem cells. However, many basic questions still remain to be addressed, including the precise mechanism by which BMP4-SMAD signaling activates Prdm1 and Prdm14 expression, the molecular nature of the competence of the epiblast cells for the germ cell fate and how WNT3 signaling would be involved in it, the biochemical mechanisms of the action of PRDM1 and PRDM14, and the precise mechanism and functional significance of the epigenetic reprogramming in PGCs after their specification. Investigations into some of these questions will require a precise in vitro PGC induction system.

There still exists only very limited information regarding the mechanism of germ cell specification in other mammals, including humans. Although the characteristics of pre-implantation development are similar between mice and humans, those of the early post-implantation development, including gastrulation, are somewhat different (Sadler 2010). In mice, the most proximal epiblast cells maintain direct contact with the extraembryonic ectoderm cells from E5.0 to E6.5, and receive a BMP4 signal from these cells to adopt the germ cell fate. In humans, before gastrulation, at around day 8 of development, some of the pluripotent epiblast cells form amnioblasts, and there appears a cavity, called the amniotic cavity, between the amnioblast and the epiblast (Sadler 2010; Fig. 5). Accordingly, the amnioblast cells directly contact the cytotrophoblast cells, and, during the second week of development, most of the epiblast cells become separated from the trophoblast layer by the amnion and the amniotic cavity (Sadler 2010; Fig. 5). There has been no information in terms of which part of the epiblast acquires the germ cell fate or which tissue emits the key signal for this fate. It is at the end of the third week that PGCs are detected by their high AP activity in the wall of the yolk sac at the base of the allantois and future umbilical cord (Sadler 2010). Clearly, it is very difficult to fully investigate the mechanism of germ cell specification in human embryos due to the difficulty of obtaining the appropriate materials, and for ethical reasons. Therefore, to gain further insight into the precise mechanism of PGC specification in humans, it will be important to investigate the mechanism of PGC specification in mammals whose early post-implantation development is similar to that of humans, such as the rabbit (Viebahn et al. 1995). The accumulation of knowledge regarding the PGC specification in mice should provide a basis for such investigations.

On the other hand, studies involving human ES and iPS cells have already shown that it is possible to induce human PGC-like cells from human ES and iPS cells (Clark et al. 2004, Kee et al. 2006, 2009, Tilgner et al. 2008, Bucay et al. 2009, Park et al. 2009). Many of these studies involve random differentiation of ES cells by EB

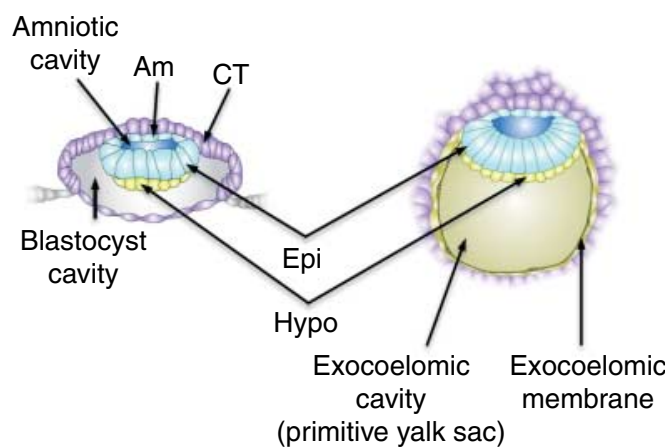

8 days

9 days

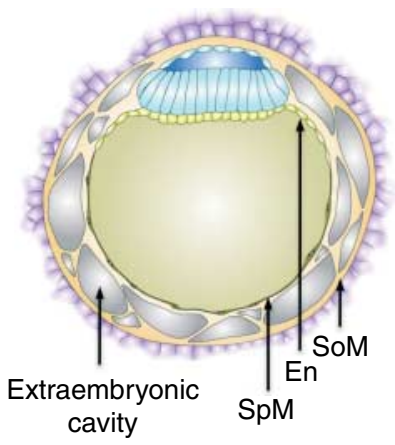

12 days

Figure 5 Schematic representation of the early post-implantation development of human embryos. The implanted human embryo at around 8 days of development consists of a syncytiotrophoblast (not shown), cytotrophoblast (CT, purple), epiblast (Epi, blue), and hypoblast (Hypo, yellow). In contrast to the mouse embryo, the epiblast cells at this stage have already formed an amnioblast (Am), and an amniotic cavity appears between the epiblast and Am. At around 12 days of development, just prior to the onset of gastrulation, the epiblast cells are no longer in direct contact with the CTs. Epi, epiblast; Hypo, hypoblast; Am, amnioblast; CT, cytotrophoblast; En, extraembryonic endoderm; SpM, extraembryonic splanchnopleuric mesoderm; SoM, extraembryonic somatopleuric mesoderm. 
formation and subsequent detection of PGC-like cells by PGC markers identified in mice. It has also been shown that the addition of BMP4 in culture substantially increases the ratio of the appearance of PGC-like cells (Kee et al. 2006, 2009). Although the demonstration of the function of induced human PGC-like cells will necessarily be made difficult by ethical considerations, an ES cell-based PGC formation, if properly performed, would provide invaluable opportunities to improve our understanding of germ cell development in humans. Continued detailed investigations into the mechanism of germ cell development in mice, as well as studies involving other mammals and human ES and iPS cells, should provide a critical foundation for a precise mechanistic understanding of the propagation of our genome both under normal and diseased conditions.

\section{Declaration of interest}

The authors declare that there is no conflict of interest that could be perceived as prejudicing the impartiality of this review.

\section{Funding}

This study was supported in part by a Grant-in-Aid from the Ministry of Education, Culture, Sports, Science, and Technology of Japan by JST, CREST, and by the Takeda Science Foundation.

\section{References}

Ancelin K, Lange UC, Hajkova P, Schneider R, Bannister AJ, Kouzarides T \& Surani MA 2006 Blimp1 associates with Prmt5 and directs histone arginine methylation in mouse germ cells. Nature Cell Biology 8 623-630.

Arnold SJ \& Robertson EJ 2009 Making a commitment: cell lineage allocation and axis patterning in the early mouse embryo. Nature Reviews. Molecular Cell Biology 10 91-103.

Assou S, Le Carrour T, Tondeur S, Strom S, Gabelle A, Marty S, Nadal L, Pantesco V, Reme T, Hugnot JP et al. 2007 A meta-analysis of human embryonic stem cells transcriptome integrated into a web-based expression atlas. Stem Cells 25 961-973.

Auman HJ, Nottoli T, Lakiza O, Winger Q, Donaldson S \& Williams T 2002 Transcription factor AP-2gamma is essential in the extra-embryonic lineages for early postimplantation development. Development 129 2733-2747.

Beck AR, Miller IJ, Anderson P \& Streuli M 1998 RNA-binding protein TIAR is essential for primordial germ cell development. PNAS 95 2331-2336.

Bedford MT \& Richard S 2005 Arginine methylation an emerging regulator of protein function. Molecular Cell 18 263-272.

Bostick M, Kim JK, Esteve PO, Clark A, Pradhan S \& Jacobsen SE 2007 UHRF1 plays a role in maintaining DNA methylation in mammalian cells. Science 317 1760-1764.

Bowles J \& Koopman P 2007 Retinoic acid, meiosis and germ cell fate in mammals. Development 134 3401-3411.

Brons IG, Smithers LE, Trotter MW, Rugg-Gunn P, Sun B, Chuva de Sousa Lopes SM, Howlett SK, Clarkson A, Ahrlund-Richter L, Pedersen RA et al. 2007 Derivation of pluripotent epiblast stem cells from mammalian embryos. Nature 448 191-195.
Bucay N, Yebra M, Cirulli V, Afrikanova I, Kaido T, Hayek A \& Montgomery AM 2009 A novel approach for the derivation of putative primordial germ cells and Sertoli cells from human embryonic stem cells. Stem Cells 27 68-77.

Buehr M, McLaren A, Bartley A \& Darling S 1993 Proliferation and migration of primordial germ cells in We/We mouse embryos. Developmental Dynamics 198 182-189.

Bussing I, Slack FJ \& Grosshans H 2008 let-7 microRNAs in development, stem cells and cancer. Trends in Molecular Medicine 14 400-409.

Calame KL, Lin KI \& Tunyaplin C 2003 Regulatory mechanisms that determine the development and function of plasma cells. Annual Review of Immunology 21 205-230.

Cao R \& Zhang Y 2004 The functions of E(Z)/EZH2-mediated methylation of lysine 27 in histone H3. Current Opinion in Genetics \& Development 14 155-164.

Chambers I, Silva J, Colby D, Nichols J, Nijmeijer B, Robertson M, Vrana J, Jones K, Grotewold L \& Smith A 2007 Nanog safeguards pluripotency and mediates germline development. Nature 450 1230-1234.

Clark AT, Bodnar MS, Fox M, Rodriquez RT, Abeyta MJ, Firpo MT \& Pera RA 2004 Spontaneous differentiation of germ cells from human embryonic stem cells in vitro. Human Molecular Genetics 13 727-739.

Covello KL, Kehler J, Yu H, Gordan JD, Arsham AM, Hu CJ, Labosky PA, Simon MC \& Keith B 2006 HIF-2alpha regulates Oct-4: effects of hypoxia on stem cell function, embryonic development, and tumor growth. Genes and Development 20 557-570.

De Miguel MP, Cheng L, Holland EC, Federspiel MJ \& Donovan PJ 2002 Dissection of the c-Kit signaling pathway in mouse primordial germ cells by retroviral-mediated gene transfer. PNAS 99 10458-10463.

Dolci S, Williams DE, Ernst MK, Resnick JL, Brannan Cl, Lock LF, Lyman SD, Boswell HS \& Donovan PJ 1991 Requirement for mast cell growth factor for primordial germ cell survival in culture. Nature $\mathbf{3 5 2}$ 809-811.

Eckert D, Buhl S, Weber S, Jager R \& Schorle H 2005 The AP-2 family of transcription factors. Genome Biology 6246.

Edson MA, Nagaraja AK \& Matzuk MM 2009 The mammalian ovary from genesis to revelation. Endocrine Reviews 30 624-712.

Extavour CG \& Akam M 2003 Mechanisms of germ cell specification across the metazoans: epigenesis and preformation. Development 130 5869-5884.

Geijsen N, Horoschak M, Kim K, Gribnau J, Eggan K \& Daley GQ 2004 Derivation of embryonic germ cells and male gametes from embryonic stem cells. Nature 427 148-154.

Ginsburg M, Snow MH \& McLaren A 1990 Primordial germ cells in the mouse embryo during gastrulation. Development 110 521-528.

Gu Y, Runyan C, Shoemaker A, Surani A \& Wylie C 2009 Steel factor controls primordial germ cell survival and motility from the time of their specification in the allantois, and provides a continuous niche throughout their migration. Development 136 1295-1303.

Guo G, Yang J, Nichols J, Hall JS, Eyres I, Mansfield W \& Smith A 2009 KIf4 reverts developmentally programmed restriction of ground state pluripotency. Development 136 1063-1069.

Hajkova P, Erhardt S, Lane N, Haaf T, El-Maarri O, Reik W, Walter J \& Surani MA 2002 Epigenetic reprogramming in mouse primordial germ cells. Mechanisms of Development 117 15-23.

Handel MA \& Schimenti JC 2010 Genetics of mammalian meiosis: regulation, dynamics and impact on fertility. Nature Reviews. Genetics 11 124-136.

Hayashi K \& Surani MA 2009 Self-renewing epiblast stem cells exhibit continual delineation of germ cells with epigenetic reprogramming in vitro. Development 136 3549-3556.

Horsley V, O'Carroll D, Tooze R, Ohinata Y, Saitou M, Obukhanych T, Nussenzweig M, Tarakhovsky A \& Fuchs E 2006 Blimp1 defines a progenitor population that governs cellular input to the sebaceous gland. Cell 126 597-609.

Hu M, Yao J, Cai L, Bachman KE, van den Brule F, Velculescu V \& Polyak K 2005 Distinct epigenetic changes in the stromal cells of breast cancers. Nature Genetics 37 899-905.

Hubner K, Fuhrmann G, Christenson LK, Kehler J, Reinbold R, De La Fuente R, Wood J, Strauss JF III, Boiani M \& Scholer HR 2003 Derivation of oocytes from mouse embryonic stem cells. Science $\mathbf{3 0 0}$ 1251-1256. 
Kallies A, Hawkins ED, Belz GT, Metcalf D, Hommel M, Corcoran LM, Hodgkin PD \& Nutt SL 2006 Transcriptional repressor Blimp-1 is essentia for T cell homeostasis and self-tolerance. Nature Immunology 7 466-474

Kanatsu-Shinohara M, Takehashi M \& Shinohara T 2008 Brief history, pitfalls, and prospects of mammalian spermatogonial stem cell research. Cold Spring Harbor Symposia on Quantitative Biology 73 17-23.

Kee K, Gonsalves JM, Clark AT \& Pera RA 2006 Bone morphogenetic proteins induce germ cell differentiation from human embryonic stem cells. Stem Cells and Development 15 831-837.

Kee K, Angeles VT, Flores M, Nguyen HN \& Reijo Pera RA 2009 Human DAZL, DAZ and BOULE genes modulate primordial germ-cell and haploid gamete formation. Nature 462 222-225.

Kehler J, Tolkunova E, Koschorz B, Pesce M, Gentile L, Boiani M, Lomeli H, Nagy A, McLaughlin KJ, Scholer HR et al. 2004 Oct4 is required for primordial germ cell survival. EMBO Reports 5 1078-1083.

Kemp C, Willems E, Abdo S, Lambiv L \& Leyns L 2005 Expression of all Wnt genes and their secreted antagonists during mouse blastocyst and postimplantation development. Developmental Dynamics 233 1064-1075.

Klose RJ, Kallin EM \& Zhang Y 2006 JmjC-domain-containing proteins and histone demethylation. Nature Reviews. Genetics 7 715-727.

Kurimoto K, Yabuta Y, Ohinata Y, Shigeta M, Yamanaka K \& Saitou M 2008 Complex genome-wide transcription dynamics orchestrated by Blimp1 for the specification of the germ cell lineage in mice. Genes and Development 22 1617-1635.

Labosky PA, Barlow DP \& Hogan BL 1994 Mouse embryonic germ (EG) cell lines: transmission through the germline and differences in the methylation imprint of insulin-like growth factor 2 receptor (Igf2r) gene compared with embryonic stem (ES) cell lines. Development 120 3197-3204.

Lawson KA \& Hage WJ 1994 Clonal analysis of the origin of primordial germ cells in the mouse. Ciba Foundation Symposia 182 68-84.

Liu P, Wakamiya M, Shea MJ, Albrecht U, Behringer RR \& Bradley A 1999 Requirement for Wnt3 in vertebrate axis formation. Nature Genetics 22 361-365.

Magnusdottir E, Kalachikov S, Mizukoshi K, Savitsky D, IshidaYamamoto A, Panteleyev AA \& Calame K 2007 Epidermal terminal differentiation depends on B lymphocyte-induced maturation protein-1. PNAS 104 14988-14993.

Martins GA, Cimmino L, Shapiro-Shelef $M$, Szabolcs M, Herron A, Magnusdottir E \& Calame K 2006 Transcriptional repressor Blimp-1 regulates T cell homeostasis and function. Nature Immunology 7 457-465.

Matsui Y, Toksoz D, Nishikawa S, Williams D, Zsebo K \& Hogan BL 1991 Effect of Steel factor and leukaemia inhibitory factor on murine primordial germ cells in culture. Nature 353 750-752.

Matsui Y, Zsebo K \& Hogan BL 1992 Derivation of pluripotential embryonic stem cells from murine primordial germ cells in culture. Cell $\mathbf{7 0}$ 841-847.

McCoshen JA \& McCallion DJ 1975 A study of the primordial germ cells during their migratory phase in Steel mutant mice. Experientia 31 589-590.

McLaren A 2003 Primordial germ cells in the mouse. Developmental Biology 262 1-15.

Mintz B \& Russell ES 1957 Gene-induced embryological modifications of primordial germ cells in the mouse. Journal of Experimental Zoology 134 207-237.

Murry CE \& Keller G 2008 Differentiation of embryonic stem cells to clinically relevant populations: lessons from embryonic development. Cell 132 661-680.

Nayernia K, Nolte J, Michelmann HW, Lee JH, Rathsack K, Drusenheimer N, Dev A, Wulf G, Ehrmann IE, Elliott DJ et al. 2006 In vitro-differentiated embryonic stem cells give rise to male gametes that can generate offspring mice. Developmental Cell 11 125-132.

Nie K, Gomez M, Landgraf P, Garcia JF, Liu Y, Tan LH, Chadburn A, Tuschl T, Knowles DM \& Tam W 2008 MicroRNA-mediated downregulation of PRDM1/Blimp-1 in Hodgkin/Reed-Sternberg cells: a potential pathogenetic lesion in Hodgkin lymphomas. American Journal of Pathology 173 242-252.

Nishikawa N, Toyota $M$, Suzuki H, Honma T, Fujikane T, Ohmura T, Nishidate T, Ohe-Toyota M, Maruyama R, Sonoda T et al. 2007 Gene amplification and overexpression of PRDM14 in breast cancers. Cancer Research 67 9649-9657.
Niwa H 2007 How is pluripotency determined and maintained? Development 134 635-646.

Ohinata Y, Payer B, O'Carroll D, Ancelin K, Ono Y, Sano M, Barton SC, Obukhanych T, Nussenzweig M, Tarakhovsky A et al. 2005 Blimp1 is a critical determinant of the germ cell lineage in mice. Nature $\mathbf{4 3 6}$ 207-213.

Ohinata Y, Ohta H, Shigeta M, Yamanaka K, Wakayama T \& Saitou M 2009 A signaling principle for the specification of the germ cell lineage in mice. Cell 137 571-584.

Okamura D, Tokitake Y, Niwa H \& Matsui Y 2008 Requirement of Oct3/4 function for germ cell specification. Developmental Biology 317 576-584.

Okita K, Ichisaka T \& Yamanaka S 2007 Generation of germline-competent induced pluripotent stem cells. Nature 448 313-317.

Park IH, Zhao R, West JA, Yabuuchi A, Huo H, Ince TA, Lerou PH, Lensch MW \& Daley GQ 2008 Reprogramming of human somatic cells to pluripotency with defined factors. Nature 451 141-146.

Park TS, Galic Z, Conway AE, Lindgren A, van Handel BJ, Magnusson M, Richter L, Teitell MA, Mikkola HK, Lowry WE et al. 2009 Derivation of primordial germ cells from human embryonic and induced pluripotent stem cells is significantly improved by coculture with human fetal gonadal cells. Stem Cells 27 783-795.

Pesce M, Farrace MG, Piacentini M, Dolci S \& De Felici M 1993 Stem cell factor and leukemia inhibitory factor promote primordial germ cell survival by suppressing programmed cell death (apoptosis). Development 118 1089-1094.

Robertson EJ, Charatsi I, Joyner CJ, Koonce $\mathrm{CH}$, Morgan M, Islam A, Paterson C, Lejsek E, Arnold SJ, Kallies A et al. 2007 Blimp1 regulates development of the posterior forelimb, caudal pharyngeal arches, heart and sensory vibrissae in mice. Development 134 4335-4345.

Runyan C, Schaible K, Molyneaux K, Wang Z, Levin L \& Wylie C 2006 Steel factor controls midline cell death of primordial germ cells and is essential for their normal proliferation and migration. Development 133 4861-4869.

Sadler TW 2010 Langman's Medical Embryology. Baltimore/Philadelphia: Lippincott Williams \& Wilkins.

Saitou M 2009a Germ cell specification in mice. Current Opinion in Genetics \& Development 19 386-395.

Saitou M 2009b Specification of the germ cell lineage in mice. Frontiers in Bioscience 14 1068-1087.

Saitou M, Barton SC \& Surani MA 2002 A molecular programme for the specification of germ cell fate in mice. Nature 418 293-300.

Sasaki H \& Matsui Y 2008 Epigenetic events in mammalian germ-cell development: reprograming and beyond. Nature Reviews. Genetics 9 129-140.

Seki Y, Hayashi K, Itoh K, Mizugaki M, Saitou M \& Matsui Y 2005 Extensive and orderly reprogramming of genome-wide chromatin modifications associated with specification and early development of germ cells in mice. Developmental Biology 278 440-458.

Seki Y, Yamaji M, Yabuta Y, Sano M, Shigeta M, Matsui Y, Saga $Y$, Tachibana M, Shinkai Y \& Saitou M 2007 Cellular dynamics associated with the genome-wide epigenetic reprogramming in migrating primordial germ cells in mice. Development 134 2627-2638.

Seydoux G \& Braun RE 2006 Pathway to totipotency: lessons from germ cells. Cell 127 891-904.

Sharif J, Muto M, Takebayashi S, Suetake I, Iwamatsu A, Endo TA, Shinga J, Mizutani-Koseki Y, Toyoda T, Okamura K et al. 2007 The SRA protein Np95 mediates epigenetic inheritance by recruiting Dnmt1 to methylated DNA. Nature 450 908-912.

Shi Y 2007 Histone lysine demethylases: emerging roles in development, physiology and disease. Nature Reviews. Genetics 8 829-833.

de Sousa Lopes SM, Roelen BA, Monteiro RM, Emmens R, Lin HY, Li E, Lawson KA \& Mummery CL 2004 BMP signaling mediated by ALK2 in the visceral endoderm is necessary for the generation of primordial germ cells in the mouse embryo. Genes and Development 18 1838-1849.

Stallock J, Molyneaux K, Schaible K, Knudson CM \& Wylie C 2003 The pro-apoptotic gene Bax is required for the death of ectopic primordial germ cells during their migration in the mouse embryo. Development 130 6589-6597. 
Tachibana M, Ueda J, Fukuda M, Takeda N, Ohta T, Iwanari H, Sakihama T, Kodama T, Hamakubo T \& Shinkai Y 2005 Histone methyltransferases G9a and GLP form heteromeric complexes and are both crucial for methylation of euchromatin at H3-K9. Genes and Development 19 815-826.

Takahashi K \& Yamanaka S 2006 Induction of pluripotent stem cells from mouse embryonic and adult fibroblast cultures by defined factors. Cell 126 663-676.

Takahashi K, Tanabe K, Ohnuki M, Narita M, Ichisaka T, Tomoda K \& Yamanaka S 2007 Induction of pluripotent stem cells from adult human fibroblasts by defined factors. Cell 131 861-872.

Tesar PJ, Chenoweth JG, Brook FA, Davies TJ, Evans EP, Mack DL, Gardner RL \& McKay RD 2007 New cell lines from mouse epiblast share defining features with human embryonic stem cells. Nature $\mathbf{4 4 8}$ 196-199.

Tilgner K, Atkinson SP, Golebiewska A, Stojkovic M, Lako M \& Armstrong L 2008 Isolation of primordial germ cells from differentiating human embryonic stem cells. Stem Cells 26 3075-3085.

Toyooka Y, Tsunekawa N, Akasu R \& Noce T 2003 Embryonic stem cells can form germ cells in vitro. PNAS 100 11457-11462.

Tsuda M, Sasaoka Y, Kiso M, Abe K, Haraguchi S, Kobayashi S \& Saga Y 2003 Conserved role of nanos proteins in germ cell development. Science 301 1239-1241.

Tsuneyoshi N, Sumi T, Onda H, Nojima H, Nakatsuji N \& Suemori H 2008 PRDM14 suppresses expression of differentiation marker genes in human embryonic stem cells. Biochemical and Biophysical Research Communications $\mathbf{3 6 7} 899-905$.

Viebahn C, Mayer B \& Hrabe de Angelis M 1995 Signs of the principle body axes prior to primitive streak formation in the rabbit embryo. Anatomy and Embryology 192 159-169.

Vincent SD, Dunn NR, Sciammas R, Shapiro-Shalef M, Davis MM, Calame K, Bikoff EK \& Robertson EJ 2005 The zinc finger transcriptional repressor Blimp1/Prdm1 is dispensable for early axis formation but is required for specification of primordial germ cells in the mouse. Development 132 1315-1325.

Weber S, Eckert D, Nettersheim D, Gillis AJ, Schafer S, Kuckenberg P, Ehlermann J, Werling U, Biermann K, Looijenga LH et al. 2010 Critical function of AP-2 gamma/TCFAP2C in mouse embryonic germ cell maintenance. Biology of Reproduction 82 214-223.
Werling U \& Schorle H 2002 Transcription factor gene AP-2 gamma essential for early murine development. Molecular and Cellular Biology 22 3149-3156.

Wernig M, Meissner A, Foreman R, Brambrink T, Ku M, Hochedlinger K, Bernstein BE \& Jaenisch R 2007 In vitro reprogramming of fibroblasts into a pluripotent ES-cell-like state. Nature 448 318-324.

West JA, Viswanathan SR, Yabuuchi A, Cunniff K, Takeuchi A, Park IH, Sero JE, Zhu H, Perez-Atayde A, Frazier AL et al. 2009 A role for Lin28 in primordial germ-cell development and germ-cell malignancy. Nature 460 909-913.

Yabuta Y, Kurimoto K, Ohinata Y, Seki Y \& Saitou M 2006 Gene expression dynamics during germline specification in mice identified by quantitative single-cell gene expression profiling. Biology of Reproduction $\mathbf{7 5}$ 705-716.

Yamaguchi S, Kurimoto K, Yabuta Y, Sasaki H, Nakatsuji N, Saitou M \& Tada T 2009 Conditional knockdown of Nanog induces apoptotic cell death in mouse migrating primordial germ cells. Development 136 4011-4020.

Yamaji M, Seki Y, Kurimoto K, Yabuta Y, Yuasa M, Shigeta M, Yamanaka K, Ohinata Y \& Saitou M 2008 Critical function of Prdm14 for the establishment of the germ cell lineage in mice. Nature Genetics $\mathbf{4 0}$ 1016-1022.

Yeom YI, Fuhrmann G, Ovitt CE, Brehm A, Ohbo K, Gross M, Hubner K \& Scholer HR 1996 Germline regulatory element of Oct-4 specific for the totipotent cycle of embryonal cells. Development 122 881-894.

Youngren KK, Coveney D, Peng X, Bhattacharya C, Schmidt LS, Nickerson ML, Lamb BT, Deng JM, Behringer RR, Capel B et al. 2005 The Ter mutation in the dead end gene causes germ cell loss and testicular germ cell tumours. Nature 435 360-364.

Yu J, Vodyanik MA, Smuga-Otto K, Antosiewicz-Bourget J, Frane JL, Tian S, Nie J, Jonsdottir GA, Ruotti V, Stewart R et al. 2007 Induced pluripotent stem cell lines derived from human somatic cells. Science $\mathbf{3 1 8}$ 1917-1920.

Received 22 January 2010

First decision 23 February 2010

Accepted 6 April 2010 